\title{
DIFFERENTIAL DIAGNOSIS OF BENIGN TUMORS OF BONE RICH IN GIANT CELLS, BASED ON IMMUNOHISTOCHEMISTRY
}

doi: 10.2478/rojost-2018-0060

\author{
L. Ali, ${ }^{1,3}$ D.M. Pop ${ }^{2,3}$, C.I. Stoica ${ }^{2,3}$ \\ "Victor Babeş" National Institute of Research and Development in the Biomedical Sciences and Biomedical \\ Sciences, Bucharest, Romania \\ 2 "Foişor" Clinical Orthopaedic Hospital, Bucharest, Romania \\ 3"Carol Davila" University of Medicine and Pharmacy, Bucharest, Romania
}

Introduction. The aim of this study was to investigate several cases of giant cell tumor of bone (TCG), chondroblastoma and aneurysmal bone cyst (ABC) by immunohistochemistry (IHC) with a panel of markers: p63, S-100, CD68, CD56, DOG1, Galectin-1, D2-40, CD34, CD45 and ki-67, some of which proved to be specific for a certain entity.

Material and methods. The cases were retrospectively selected from cases processed in our facility where the surgical excision material was histopathologically analyzed in optical microscopy using the usual staining hematoxylin and eosin. The immunohistochemistry exam was performed at INCDVB by indirect method avidin-biotin-peroxidase complex (C-DAB) with polyclonal antibodies (Dako) for S100; CD56, p63, DOG1, CD34, D2-40, CD45, ki67, and Galectin1 were performed in 14 cases out of which 10 were TGC, 3 chondroblastomas, and one primary ABC.

Results. Five out of ten TGC investigated showed p63 diffuse nuclear expression in the mononuclear cell. None of the 3 cases of chondroblastoma or ABC-primary or secondary (associated with the above mentioned tumors), expressed p63. Other variables, non-specifically encountered in all of the tumors, were emphasized by $\mathrm{CD}_{34}$ (vascular network), ki-67 (index of proliferation), $\mathrm{CD}_{45}$ (inflammatory infiltrate). DOG1 expression was found positive in all chondroblastoma cases.

Conclusions. p63 proved to be a useful biomarker in differentiating giant cell tumor of bone from central giant cell granuloma and other giant cell-rich tumors, especially chondroblastoma and $A B C$. Chondroblastoma was particularly rewarding in this investigation by demonstrating the dual immunohistochemical phenotype of the neoplastic cells, both chondroblastic (S-100, D2-40) and osteoblastic (CD56 and Galectin -1). DOG1, which is mainly used for the diagnostic of GIST, was found by many to be also specific, in the proper morphopathological context, for chondroblastoma. Keywords: giant cell tumors of bone, p63, DOG1 